[0212-7199 (2004) 21: 12; pp 593-596] ANALES DE MEDICINA INTERNA Copyright (C) 2004 ARAN EDICIONES, S.L.

AN. MED INTERNA (Madrid) Vol. 21, N. $^{\circ} 12$, pp. 593-596, 2004

\title{
Enfermedad de Erdheim-Chester e histiocitosis de células de Langerhans. ¿Una asociación fortuita?
}

\author{
A. SIMIELE NARVARTE, F. NOVOA SANJURJO, N. GÓMEZ RODRÍGUEZ1, \\ I. ANTÓN BADIOLA ${ }^{2}$
}

Servicios de Hematología, ${ }^{1}$ Reumatología y ${ }^{2}$ Anatomía Patológica. Centro Médico

POVISA. Vigo. Pontevedra

\begin{abstract}
ERDHEIM-CHESTER DISEASE AND LANGERHANS HISTIOCYTOSIS. A FORTUITOUS ASSOCIATION?
\end{abstract}

\section{RESUMEN}

La enfermedad de Erdheim-Chester es una histiocitosis sistémica de origen desconocido, histológicamente distinta de la histiocitosis de células de Langerhans, que se caracteriza por lesiones óseas esclerosantes de distribución simétrica, con predominio en las diáfisis y metáfisis de huesos largos. No está clasificada dentro de las histiocitosis malignas, no obstante, su curso clínico suele ser agresivo, con escasa respuesta a los diversos tratamientos ensayados. Excepcionalmente, se han descrito casos con hallazgos anatomopatológicos de la enfermedad de ErdheimChester asociados a la presencia de histiocitos de Langerhans.

Presentamos un nuevo paciente en el que se identificaron cambios radiográficos compatibles con enfermedad de Erdheim-Chester pero cuyo estudio histológico mostró una histiocitosis de células de Langerhans y cursó con ostealgias, mialgias, diabetes insípida, exoftalmos, lesiones óseas esclerosantes bilaterales y simétricas así como un síndrome cerebeloso.

PALABRAS CLAVE: Enfermedad de Erdheim-Chester. Histiocitosis de células de Langerhans. Diabetes insípida. Síndrome cerebeloso.

\begin{abstract}
Erdheim-Chester disease is an idiopathic systemic histiocytosis hystologically different to Langerhans histiocytosis which is characterized by symmetric sclerotic bone lesions, predominantly affecting the diaphyseal and metaphyseal areas of large bones. It is not classified within malignant histicytosis, but the clinical course tends to be agressive with a poor reponse to different schedules of treatment. In rare cases the association of both entities has been reported: Erdheim-Chester disease and Langerhans histiocytosis.

We present a new case with histological data of both histiocytosis whose clinical course included bone and muscle pain, insipidus diabetes exophthalmos, bilateral symmetrical sclerosing bone lesions and a cerebellar syndrome.
\end{abstract}

KEY WORDS: Erdheim-Chester disease. Langerhans histiocytosis. Insipidus diabetes. Cerebellar syndrome.

Simiele Narvarte A, Novoa Sanjurjo F, Gómez Rodríguez. N, Antón Badiola I. Enfermedad de Erdheim-Chester e histiocitosis de células de Langerhans. ¿Una asociación fortuita? An Med Interna (Madrid) 2004; 21: 593-596.

\section{INTRODUCCIÓN}

En 1930, Chester (1) describió una forma particular de lipogranulomatosis en dos pacientes que presentaban lesiones óseas esclerosantes con tendencia a la simetría. En 1972, Jaffe (2) estudia otro caso similar y acuña el epónimo de enfermedad de Erdheim-Chester. En la actualidad se le considera una entidad infrecuente, caracterizada por la infiltración tisular mediante histiocitos cargados de lípidos que provocan lesiones óseas esclerosantes de predominio en diáfisis y metáfisis, sobre todo en las extremidades inferiores (3-5). Se trata de un proceso sistémico en el que, entre otras, se han reseñado manifestaciones endocrinológicas $(6,7)$, oftalmológicas (8$10)$, cardiovasculares (11-13), neurológicas (14-16), urinarias (17-19) y pulmonares (20) .

\section{CASO APORTADO}

Varón de 54 años, con antecedentes de hepatitis B, diagnosticado de diabetes insípida desde los 30 años, que fue remitido a reumatología por astenia, ostealgias y mialgias, más intensas en muslos y cintura pélvica, sobre todo durante la deambulación. No se recogían antecedentes familiares de interés y únicamente se encontraba a tratamiento con desmopresina.

Trabajo aceptado: 1 de junio de 2004

Correspondencia: Norberto Gómez Rodríguez. Unidad de Reumatología. Colón, 28 Clínica. C/ Colón, 28-1². 36201 Vigo. Pontevedra. e-mail: colon28@povisa.es 
El paciente presentaba un buen estado general y únicamente destacaba un exoftalmos bilateral, sin xantelasmas. Todas las articulaciones periféricas estaban libres y tanto la auscultación cardiopulmonar como el examen neurológico y la palpación abdominal fueron normales.

Entre los parámetros de laboratorio cabía reseñar una leve anemia microcitica e hipocrómica (Hb: $124 \mathrm{~g} / \mathrm{L}$, Htc: 0,39 L/L), VSG: $81 \mathrm{~mm} / 1^{\mathrm{a}} \mathrm{h}$, proteína C reactiva: $10,1 \mathrm{mg} / \mathrm{L}$. Las determinaciones de transaminasas, gammaglutamiltranspeptidasa, fosfatasa alcalina, bilirrubina, creatinina, urea, glucosa, colesterol, triglicéridos, calcio, fósforo, vitamina B12, folato, hierro, Ferritina, lácticodehidrogenasa, proteinograma, inmunoglobulinas, hormonas tiroideas, antígeno prostático específico, prolactina, testosterona, dentro de los valores normales. El sedimento urinario fue normal y en la electroforesis en suero y orina de 24 horas no se identificó componente monoclonal. Los siguientes parámetros fueron negativos: factor reumatoide (látex), autoanticuerpos (antinucleares, anti-DNA, anticardiolipina, anti-ENA, ANCA), serología luética reagínica y treponémica, así como los marcadores de la hepatitis C. La electromiografía de los músculos de las extremidades inferiores fue normal.

Las radiografía de tórax mostró un engrosamiento pulmonar apical bilateral, sin otras alteraciones. Las radiografías de pelvis y huesos largos de las extremidades inferiores mostraron engrosamiento cortical y alternancia de lesiones escleróticas y osteolíticas con afectación de áreas diafisarias y metafisarias. Ambos iliacos estaban involucrados (Fig. 1). La gammagrafía ósea con $\mathrm{Tc}^{99 \mathrm{~m}}$ evidenció hipercaptación del trazador localizada en ambas cabezas humerales y de forma difusa en iliacos, fémures y tibias. En la resonancia de pelvis y fémures se observaron áreas moteadas de hiposeñal en T2 en las cavidades medulares, respetando las epífisis (Fig. 2). En la biopsia de la médula ósea (cresta iliaca) no se halló presencia de células histiocitoides ni otras anomalías. En la biopsia ósea (fémur derecho) destacaba un hueso esclerótico con gran componente fibrótico, aumento de las fibras de colágeno e infiltración por polimorfonucleares eosinófilos y células de Langerhans. En el estudio inmunohistoquímico, estos histiocitos mostraron positividad para el CD1a y el antígeno S100 fue negativo.

Se adoptó una actitud expectante y se mantuvo tratamiento con analgésicos para los dolores de la cintura pélvica y muslos, cuya intensidad fue discreta o moderada. Durante el sexto año de seguimiento consultó por presentar trastornos en la marcha y en el equilibrio e impotencia. Se evidenció exoftalmos asimétrico, más acentuado en el ojo derecho, un nistagmo horizontal a la derecha, sin paresias de pares craneales, y pruebas cerebelosas positivas, siendo el resto de la exploración normal. Se realizaron nuevas determinaciones hematimétricas, bioquímicas, serológicas que no demostraron cambios respecto a las previas, excepto disminución de la VSG (58
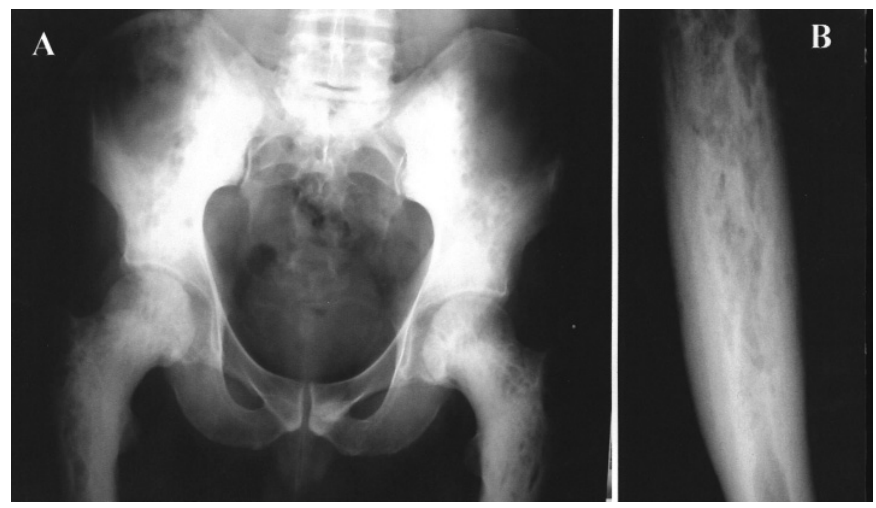

Fig. 1. A: Radiografía frontal de la pelvis del paciente en la que puede apreciarse la alternancia de áreas de hueso esclerótico con lesiones osteolíticas que involucran ambos iliacos y fémures. B: Detalle del fémur izquierdo en el que se observa un patrón patológico mixto.

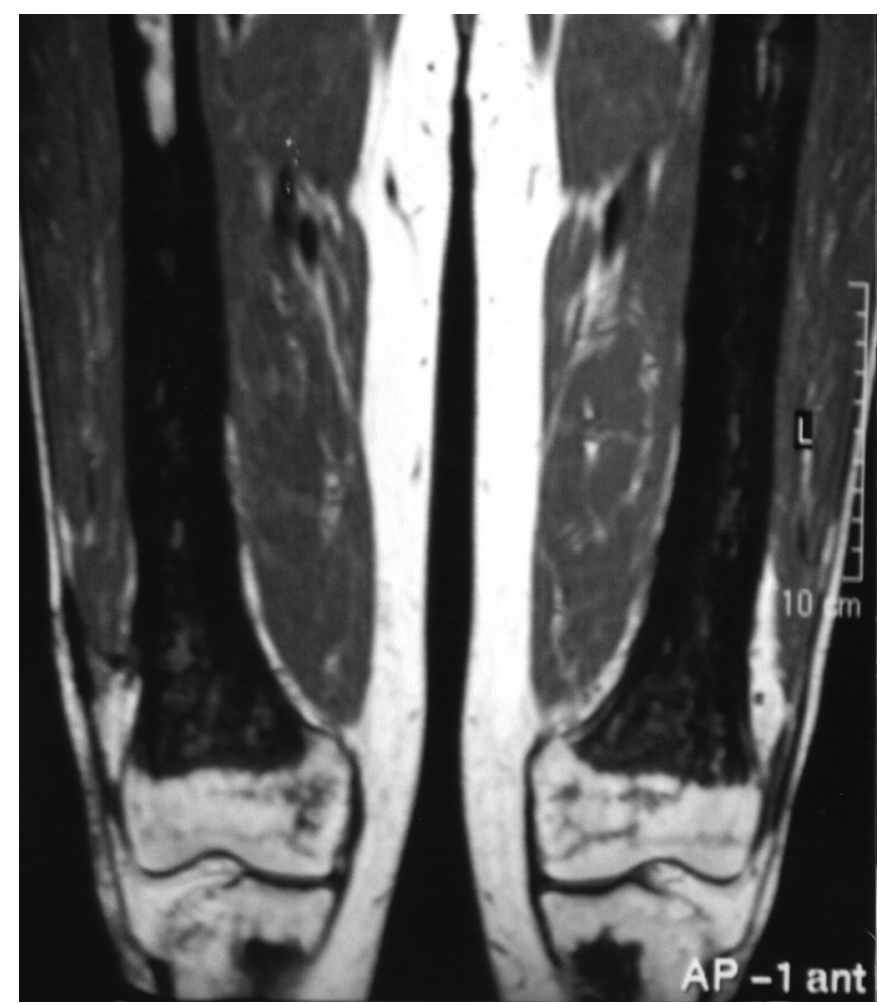

Fig. 2. Imagen coronal de resonancia magnética, ponderada en $T 2$, en la que se aprecian las alteraciones de la señal en la cavidad medular (hiposeñal moteada), excepto en las epífisis, que están respetadas.

$\mathrm{mm} / 1^{\mathrm{a} h}$ ). En la resonancia cerebral se comprobó que el exoftalmos no se asociaba a masas retroorbitarias y se identificó atrofia cortical cerebral y, sobre todo, cerebelosa. Las radiografías de huesos largos de las extremidades y pelvis no mostraron modificaciones respecto a las previas. El estudio del líquido cefalorraquídeo no demostró alteraciones. Se repitió la biopsia de médula ósea sin hallar alteraciones reseñables. Una nueva biopsia ósea (fémur izquierdo) fue similar a la previa.

El paciente permanece estable, sin que se hayan producido modificaciones sintomáticas significativas en los últimos tres años.

\section{DISCUSIÓN}

La enfermedad de Erdheim-Chester es una rara forma de histiocitosis que afecta a pacientes de todas las edades, sin preferencia por sexos, y cuya expresividad clínica oscila desde lesiones óseas asintomáticas hasta la muerte como consecuencia de complicaciones sistémicas (3). El sustrato anatomopatológico, tanto de las lesiones óseas como de las manifestaciones extraesqueléticas es la infiltración de los tejidos por células gigantes multinucleadas (células de Touton) e histiocitos cargados de lípidos en cuya membrana se detecta el antígeno CD 68 pero que, a diferencia de las células de Langerhans, no expresan el antígeno CD1a y, de modo variable, presentan el antígeno $\mathrm{S} 100(5,21)$.

Las ostealgias y las artralgias, referidas por la mitad de los pacientes, son los síntomas más prominentes y suelen localizarse en las extremidades inferiores (22). En la mitad de los casos se recogen manifestaciones extraesqueléticas (Tabla I). Pueden aparecer síntomas inespecíficos como astenia, fatiga o 


\section{TABLA I}

MANIFESTACIONES CLÍNICAS DE LA ENFERMEDAD DE ERDHEIM-CHESTER

1. Manifestaciones en el aparato locomotor

Ostealgias

Artralgias

Mialgias

2. Manifestaciones extraesqueléticas

Endocrinológicas

- Hipogonadismo hipogonadotrófico

-Diabetes insípida

- Hipopituitarismo

- Hiperprolactinemia

Oftalmológicas

-Exoftalmos uni o bilateral

-Xantelasmas

- Masas orbitarias

Cardiovasculares

-Pericarditis con o sin derrame

- Pseudotumores cardíacos

-Aortitis

Neuropsiquiátricas

- Paraparesia y paraplejia

- Síndrome cerebeloso

Genitourinarias

- Trastornos psiquiátricos

-Pseudotumores renales

- Hidronefrosis y otras formas de uropa-

tía obstructiva

-Estenosis de las arterias renales

-Síndrome nefrótico

Pulmonares

-Neumopatía intersticial

-Derrame pleural

Otorrinolaringológicas

-Sinusopatía

Otras posibles manifestaciones

-Pseudotumor mamario

-Periodontitis

-Amiloidosis

-Lesiones cutáneas

pérdida de peso. La infiltración del hipotálamo e hipófisis desencadena diversos trastornos endocrinológicos, entre los que cabe destacar la diabetes insípida, el hipogonadismo hipogonadotropo y el hipopituitarismo $(6,7)$. La infiltración de los tejidos orbitarios provoca exoftalmos uni o bilateral y cuando se produce en los párpados da lugar a xantelasmas (8-10). La infiltración y subsiguiente fibrosis retroperitoneal puede comprometer los uréteres y condicionar una hidronefrosis (18) o bien desencadenar una coartación aórtica (11). Se ha descrito la afectación pulmonar en forma de neumopatía instersticial y derrame pleural (20), así como fibrosis mediastínica, pseudotumores cardíacos (12) y pericarditis (13). La implicación del corazón y / o pericardio comporta peor pronóstico a corto plazo. Los trastornos neurológicos derivados de la infiltración (microscópica o macroscópica) del cerebelo y núcleos de la base cerebral $(14,23)$ así como los ocasionados por compro- miso de la médula espinal $(24,25)$, que pueden llegar a la paraplejia, son raros. Excepcionalmente se ve afectado el músculo esquelético (26) o las mamas (27). Como en otras enfermedades crónicas, el curso evolutivo puede complicarse con una amiloidosis secundaria (28). La evolución suele ser lenta, gradual, con tendencia al empeoramiento. En los casos con afectación visceral, la muerte suele acaecer por fallo multiorgánico.

Los hallazgos radiográficos son característicos y bilaterales. Consisten en esclerosis ósea parcheada o difusa, coalescencia del patrón trabecular, aumento del espesor de las corticales, principalmente en las metáfisis, con escasa afectación epifisaria. Los huesos largos de las extremidades inferiores, en especial el fémur y, en menor grado tibia y peroné, están implicados con mucha mayor frecuencia e intensidad que el esqueleto axial $(3,29,30)$. No obstante, se ha descrito algún caso de distribución atípica de las lesiones óseas, con afectación casi exclusiva de las vértebras y huesos pélvicos (31). Las áreas osteolíticas son expresión de la infiltración histiocitaria local y son más evidentes en los huesos planos como costillas, cráneo o las vértebras, sobre todo en las fases iniciales de la enfermedad. Posteriormente, conforme la infiltración da paso a la fibrosis regional, se desarrolla osteosclerosis. En la resonancia magnética, las imágenes ponderadas en T1 presentan una señal disminuida, mientras que las ponderadas en T2 y STIR son mixtas (zonas hiperintensas alternando con otras hipointensas). La médula ósea presenta una reducción de la señal en T1 proporcional al grado de infiltración y fibrosis. Cuando se administra gadolinio se produce un aumento de la señal en las áreas involucradas que puede prolongarse hasta dos semanas (32). La resonancia magnética es la técnica de elección cuado se sospecha afectación medular extra o intradural. Además, también es de gran utilidad para analizar la localización y la extensión de las lesiones viscerales como las del sistema genitourinario, el aparato digestivo o el sistema nervioso central (22). En un tercio de los pacientes existe afectación pulmonar. En las radiografías suelen observarse un patrón retículonodular, mientras que la tomografía computarizada muestra un ensanchamiento de los septos interlobares y de la pleura visceral así como nódulos de predominio centrolobular (32). Las gammagrafías óseas, bien con $\mathrm{T}^{\mathrm{c} 99 \mathrm{~m}}$, bien con $\mathrm{Ga} 67$ son útiles para determinar la actividad y la extensión del proceso, aunque no poseen especificidad (33).

El diagnóstico diferencial se plantea clínicamente con otras histiocitosis y con entidades nosológicas capaces de producir lesiones óseas mixtas como la enfermedad de Paget, la sarcoidosis, algunos linfomas y enfermedades de depósito como la enfermedad de Gaucher. En quienes destaca la afectación pulmonar, el espectro del diagnóstico diferencial se amplía a la malakoplaquia pulmonar, síndrome de Hermansky-Pudlak (34) y enfermedad de Rosai-Dorfman (35). El diagnóstico se confirma anatomopatológicamente. A diferencia de la enfermedad de Langerhans, los histiocitos y macrófagos de las lesiones de la enfermedad de Erdheim-Chester no poseen un núcleo indentado ni gránulos de Birbeck en el citoplasma. Otro hecho diferencial es la ausencia en la membrana celular de la proteína calciotropa S100 y de la glucoproteína CD1a.

Debido a su relativa rareza y la escasa respuesta a los diversos tratamientos ensayados, todavía no se se dispone de una pauta terapéutica consensuada en la enfermedad de 
Erdheim-Chester. Se han empleado con mayor o menor éxito esteroides, ciclosporina, azatioprina, interferón- $\alpha 2^{a}$ y citostáticos como vinblastina, vincristina, doxorubicina o ciclofosfamida $(3,32)$. La radioterapia se ha utilizado de forma empírica, pero ha tenido fracasos (36). En un paciente de 18 años en el que existía intensa infiltración orbitaria que comprometía la visión se aplicó con éxito relativo el trasplante de células madre hematopoyéticas (37).

\section{Bibliografía}

1. Chester W. Über Lipoidgranulomatose. Virchows Arch 1930-31; 279: 561-602.

2. Jaffe HL. Metabolic, degenerative and inflammatory diseases of bones and joints. Philadelphia: Lea \& Febiger, 1972. p. 535-541.

3. Veyssier-Belot C, Cacoub P, Caparros-Lefebvre D, Wechsler J, Brun B, Remy M, et al. Erdheim-Chester disease. Clinical and radiologic characteristics of 59 cases. Medicine (Balt) 1996; 75: 157-169.

4. Chetritt J, Paradis V, Dargere D, Adle-Biassette H, Maurage CA, Mussini JM, et al. Chester-Erdheim disease: a neoplastic disorder. Hum Pathol 1999; 30: 1093-1096.

5. Kenn W, Eck M, Allolio B, Jacob F, Illg A, Marx A, et al. ErdheimChester disease: evidence for a disease entity different from Langerhans cell histiocytosis? Three cases with detailed radiological and inmunohistochemical analysis. Hum Pathol 2000; 31: 734-739.

6. Tritos NA, Weinrib S, Kaye TB. Endocrine manifestations of ErdheimChester disease (a distinct form of histiocytosis). J Intern Med 1998 244: 529-535.

7. Khamseh ME, Mollanai S, Hashemi F, Rezaizadeh A, Azizi F. Erdheim-Chester syndrome, presenting as hypogonadotropic hypogonadism and diabetes insipidus. J Endocrinol Invest 2002; 25: 727-729.

8. De Palma P, Ravali L, Grisanti F, Rossi A, Marzola A. Nielsen I. Bilateral orbital involvement in Erdheim-Chester disease. Orbit 1998; 17: $97-$ 105.

9. Amrit S, Low CH, Cheah E, Tan YO. Erdheim-Chester disease: a bilateral orbital mass as an indication of systemic disease. Orbit 1999; 18: 99-104.

10. Le Goff L, Berros P, Denis D, Ridings B. Exophtalmie bilaterale - diabete insipide: la maladie d'Erdheim-Chester. J Fr Opthalmol 2002; 25: 57-61.

11. Serratrice J, Granel B, De Roux C, Pellissier JF, Swiader L, Bartoli J M et al. "Coated aorta": a new sign of Erdheim-Chester disease. J Rheumatol 2000: 27: 1550-1553.

12. Ammann P, Boesch B, Buchholz S, Genoni M, Laube I, Naegeli B, et al. Cardiac tumor due to Erdheim-Chester disease. Am J Med 2001; 111: 672-673.

13. Gupta A, Kelly B, McGuigan J . Erdheim-Chester disease with prominent pericardial involvement: clinical, radiologic, and histologic findings. Am J Med Sci 2002; 324: 96-100.

14. Wright RA, Hermann RC, Parisi JE. Neurological manifestations of Erdheim-Chester disease. J Neurol Neurosurg Psychiatry 1999; 66: 7275.

15. Oweity T, Scheithauer BW, Ching H S, Lei CMC, Wong KP. Multiple system Erdheim-Chester disease with massive hypothalamic-sellar involvement and hypopituitarism. Case report and review of the literature. J Neurosurg 2002; 96: 344-351.

16. Reithmeier T, Trost H A, Wolf S, Stoelzle A, Feiden W, Lumenta C B et al. Xanthogranulomata of the Erdheim-Chester type within the sellar region: case report. Clin Neuropathol 2002; 21: 24-28.

17. Enríquez R, Cabezuelo JB, Martínez M, Sáez J, Sirvent AE, Amoros F, et al. Nephrotic síndrome and amyloid A amiloidosis in a patient with Erdheim-Chester disease. Nephron 2000; 86: 195-196.

18. Fortman B J, Beal, D P. Erdheim-Chester disease of the retroperitoneum: a rare cause of ureteral obstruction. A J R 2001; 176: 1330-1331.

19. Yun EJ, Yeh BM, Yabes AP, Coakley FV, Kane CJ. Erdheim-Chester disease: case report and review of associated urological, radiological and histological features. J Urol 2003; 169: 1470-1471.
20. Rush WL, Andriko JA, Galateau-Salle F, Brambilla E, Brambilla C, Ziany-Bey I, et al. Pulmonary pathology of Erdheim-Chester disease. Mod Pathol 2000; 13: 747-754.

21. Al-Quran S, Reith J, Bradley J, Rimaza L. Erdheim-Chester disease: case report, PCR-based analysis of clonality, and review of literature. Mod Pathol 2002; 15: 666-672.

22. Gottlieb R, Chen A. MR findings of Erdheim-Chester disease. J Comput Assist Tomogr 2002; 26: 257-261.

23. Vital C, Bioulac-Sage P, Tison F, Rivel J, Begueret H, Gómez C, et al. Brain stem infiltration by mixed Langerhans cell histiocytosis and Chester-Erdheim disease: more than just an isolated case? Clin Exp Pathol 1999; 47: 71-76.

24. Albayram S, Kizilkilic O, Zulfikar Z, Islak C, Kocer N. Spinal dural involvement in Erdheim-Chester disease: MRI findings. Neuroradiology 2002; 44: 1004-1007.

25. Pego R, Brañas F, Martínez F, Rivas M J, Sanjuanbenito L, García M et al. Erdheim-Chester disease with spinal cord manifestations. Eur Neurol 2000; 43: 242-244.

26. Yamamoto T, Mizuno K. Erdheim-Chester disease with intramuscular lipogranuloma. Skelet Radiol 2000; 29: 227-230.

27. Ferrozzi F, Bova D, Tognini G, Zuccoli G. Pseudotumoral bilateral involvement of the breast in Erdheim-Chester disease: CT appearance. J Comput Assist Tomogr 2000; 24: 281-183.

28. Enríquez R, Cabezuelo JB, Martínez M, Sáez J, Sirvent AE, Amoros F, et al. Nephrotic síndrome and amyloid A amiloidosis in a patient with Erdheim-Chester disease. Nephron 2000; 86: 195-196.

29. Kenn W, Stabler A, Zachoval R, Zietz C, Raum W, Wittenberg G. Erdheim-Chester disease: a case report and literature overview. Eur Radiol 1999; 9: 153-158.

30. Breuil V, Brocq O, Pellegrino C, Grimaud A, Euller-Ziegler L, et al. Erdheim-Chester disease: typical radiological bone features for a rare xanthogranulomatosis. Ann Rheum Dis 2002: 61: 199-200.

31. Klieger MR, Schultz E, Elkowitz DE, Arlen M, Hajdu SI. ErdheimChester disease: a unique presentation with múltiple osteolytic lesions of the spine and pelvis that spared the appendicular skeleton. A J R 2002; 172: 429-432.

32. Shamburek RD, Brewer HB Jr, Gochuico BR. Erdheim-Chester disease: a rare multisystem histiocytic disorder associated with intersticial lung disease. Am J Med Sci 2001; 321: 66-75.

33. Franzius C, Sciuk J, Bremer C, Kempkes M, Schober O. Determination of extent and activity with radionuclide imaging in Erdheim-Chester disease. Clin Nucl Med 1999; 24: 252-255.

34. Gahl WA, Brantly M, Kaiser-Kupfer MI, Iwata F, Hazelwood S, Shotelersuk V, Duffy LF, Kuehl EM, Troendle J, Bernardini I. Genetic defects and clinical characteristics of patients with a for of oculomcutaneous albinism (Hermansky-Pudlak Syndrome). N Engl J Med 1998; 338: 1258-1264.

35. Foucar E, Rosai J, Dorfman R. Sinus histiocytosis with massive lymphadenopathy (Rosai-Dorfman disease): review of the entity. Semin Diagn Pathol 1990; 7: 19-73.

36. Mascalchi M, Nencini P, Nistri M, Sarti C, Santoni R. Failure of radiation therapy for brain involvement in Erdheim-Chester disease. J Neurooncol 2002; 59: 169-172.

37. Boissel N, Wechsler B, Leblond V. Treatment of refractory ErdheimChester disease with double autologous hematopoietic stem-cell transplantation. Ann Intern Med 2001; 135: 844-845. 